\title{
Effects of the epitaxial layer thickness on the noise properties of Schottky barrier diodes
}

\author{
G. Gomila ${ }^{\text {a) }}$ \\ Istituto Nazionale di Fisica della Materia, Dipartamento di Ingegneria dell'Innovazione, \\ Università di Lecce, Via Arnesano, I-73100 Lecce, Italy \\ O. M. Bulashenko \\ Departament de Física Fonamental, Universitat de Barcelona, Diagonal 647, E-08028 Barcelona, Spain
}

(Received 17 February 1999; accepted for publication 12 April 1999)

\begin{abstract}
An analytical theory to describe the combined effects of the epitaxial layer thickness and the ohmic contact on the noise properties of Schottky barrier diodes is presented. The theory, which provides information on both the local and the global noise properties, takes into account the finite size of the epitaxial layer and the effects of the back ohmic contact, and applies to the whole range of applied bias. It is shown that by scaling down the epitaxial layer thickness, the current regime in which the noise temperature displays a shot-noise-like behavior increases at the cost of reducing the current range in which the thermal-noise-like behavior dominates. This improvement in noise temperature is limited by the effects of the ohmic contact, which appear for large currents. The theory is formulated on general trends, allowing its application to the noise analysis of other semiconductor devices operating under strongly inhomogeneous distributions of the electric field and charge concentrations. (C) 1999 American Institute of Physics. [S0021-8979(99)04714-3]
\end{abstract}

\section{INTRODUCTION}

Schottky barrier diodes are being extensively used in different applications such as mixers and detectors, due to their good high frequency behavior. ${ }^{1}$ In recent years, for instance, THz Schottky barrier diodes have been the subject of considerable attention. ${ }^{1-6}$ Currently, the important point is how to improve their realization and technology to obtain still better results at higher frequencies. To this respect, much effort has been devoted to reduce the diode thickness, thereby reducing the parasitic resistance loses in the bulk region maintaining sufficient nonlinearity characteristics for applications. ${ }^{1}$ An important problem, in this respect, is to understand the role played by the epitaxial layer thickness and ohmic contacts on the performance of these devices. For thin epitaxial layers, to approach this problem, one needs to solve the equations for the transport and for the fluctuations, with appropriate boundary conditions on both ends of the diode. With respect to transport properties this study has been already performed. ${ }^{2,7}$ However, with respect to the noise properties the equivalent analysis is still lacking in the current literature. The aim of this article is to address this issue.

The existing noise models are usually based on the phenomenological equivalent circuit approach. ${ }^{8}$ These models consider the existence of two main noise sources: a shotnoise source associated with the junction resistance and a thermal-noise source associated with the series resistance. ${ }^{9,12}$ Sometimes, to explain some of the excess noise observed experimentally, other noise sources due to hot electrons, intervalley scattering, trapping, etc., are introduced in the same phenomenological scheme. ${ }^{3,10,11,13}$ However, for very thin epitaxial layers, the division between series and junction re-

${ }^{\text {a)} E l e c t r o n i c ~ m a i l: ~ g o m i l a @ a x p m a t . u n i l e . i t ~}$ sistances loses its meaning, since the depletion layer extends over all the diode. In such a case, the combined effect of the diode size and the nonideality of the back ohmic contact may become relevant, and the phenomenological theories may not apply.

Recently, a more advanced analytical self-consistent treatment has been proposed for the noise analysis of a Schottky diode. ${ }^{14}$ Despite of its simplicity (it is based on the drift-diffusion treatment of the carrier transport), the method is able to provide, not only the net noise parameters, such as noise temperature, voltage and current noise spectral densities, but also the local noise distributions. That is, the contribution of different parts of the diode to the net noise is obtained in an analytical form in terms of the steady-state electric-field profiles. Such an approach allowed one to conclude that the cross correlations between the two main noise sources: the shot-noise and the thermal-noise one, is also important at around flatband bias conditions, the typical operation conditions of $\mathrm{THz}$ Schottky diodes. ${ }^{1,3}$ It should be noted, that the Monte Carlo procedure, as an alternative possibility to investigate the transport and noise properties of Schottky barrier diodes, meets difficulties of poor statistics of carriers in the depletion layer, ${ }^{15}$ a problem which does not appear in the drift-diffusion framework.

In all the models mentioned above, however, the ohmic contact was assumed to be completely perfect, that is nondisturbing the local electric field and charge concentrations. In this article, based upon the thermionic-emission-diffusion framework, ${ }^{14}$ we perform an analytical analysis of the noise properties of Schottky barrier diodes when their epi-layer thickness $L$ is scaled down to submicron scale $\left(L \leqq L_{D}\right.$, with $L_{D}$ being the Debye screening length). By making use of appropriate boundary conditions for both ends of the epitaxial layer, we study the combined effects of the epitaxial layer 
thickness and the back ohmic contact on the noise properties in the whole range of applied bias. It is worth noting that the present study requires the development of a more advanced mathematical treatment than that presented previously in Ref. 14. In fact, this new treatment in itself, offers new perspectives concerning the analytical analysis of noise in other semiconductor devices.

The organization of the article is as follows. In Sec. II, we briefly review the basic equations for the description of transport and fluctuations in the thermionic-emissiondiffusion approach. In Sec. III, the steady-state spatial profiles for the electric field and carrier density, as well as the $I-V$ characteristics, are numerically obtained. In Sec. IV A, we present analytical expressions for the impedance and noise characteristics. Their local counterparts, the impedance field and the local noise distributions, are also presented. In Sec. IV B, we evaluate the derived analytical expressions for different diode thicknesses and different current operation regimes. In particular, we focus on the role played by the epitaxial layer thickness and ohmic contact on the noise properties. Finally, in Sec. V, we sum up the main contributions of the article, whereas the appendixes are devoted to some mathematical derivations.

\section{THERMIONIC EMISSION-DIFFUSION THEORY: TRANSPORT AND FLUCTUATIONS}

\section{A. Charge transport}

We are interested in modelling the low-frequency plateau of the noise spectrum corresponding to the time scale much longer than the dielectric relaxation time. Due to the frequency range chosen, the displacement current can be neglected. In the drift-diffusion approximation, combining the current and Poisson equations, the electric transport is governed by ${ }^{14,16}$

$$
D(E) \frac{d^{2} E}{d x^{2}}+v(E)\left(\frac{d E}{d x}-\frac{q}{\epsilon} N_{D}\right)=-\frac{I}{\epsilon A},
$$

where $E=E(x)$ is the electric field, $I$ the electric current, $q$ the electron charge, $\epsilon$ the dielectric permittivity, $A$ the sample cross-sectional area, $N_{D}$ the doping density, $D(E)$ the diffusion coefficient, and $v(E)$ the electron velocity.

Furthermore, the boundary condition at the metalsemiconductor rectifying contact located at $x=0$ reads $^{14}$

$$
\left.\frac{d E}{d x}\right|_{x=0}=\frac{q}{\epsilon}\left(N_{D}-n_{0}^{e q}-\frac{I}{q v_{r}^{0} A}\right),
$$

where $v_{r}^{0}$ is the recombination velocity of the contact and $n_{0}^{e q}=N_{c} e^{-q \phi_{b}^{0} / k_{B} T}$ the quasiequilibrium electron density, with $N_{c}$ being the semiconductor effective density of states, $k_{B}$ the Boltzmann constant, $T$ the lattice temperature, and $\phi_{b}^{0}$ the contact barrier height.

On the opposite side of the epitaxial layer, an ohmic contact is implemented. In the present article, we will assume an ohmic metal-semiconductor contact, although other contacts can be equally studied. For a metal-semiconductor ohmic contact, a similar equation to Eq. (2) applies,

$$
\left.\frac{d E}{d x}\right|_{x=L}=\frac{q}{\epsilon}\left(N_{D}-n_{L}^{e q}+\frac{I}{q v_{r}^{L} A}\right),
$$

where $v_{r}^{L}$ is the recombination velocity of the ohmic contact and $n_{L}^{e q}=N_{c} e^{-q \phi_{b}^{L} / k_{B} T}$ its quasiequilibrium electron density, with $\phi_{b}^{L}$ being the barrier height for the metalsemiconductor ohmic contact. The main difference between the two contacts relies in the values of the barrier height. While for the rectifying contact it is large $\left(\phi_{b}^{0} \gtrsim 15 k_{B} T / q\right)$, for ohmic contact, it is relatively small $\left(\phi_{b}^{L} \lesssim 10 k_{B} T / q\right)$. As a result, the contact resistance, which depends exponentially on the barrier height, is very large for a rectifying contact and relatively small for an ohmic contact.

\section{B. Fluctuations}

According to the thermionic-emission-diffusion model for the fluctuations introduced in Ref. 14, the noise properties of the system can be analyzed from the fluctuations of the electric field. In the Langevin approach, the fluctuations of the electric field $\delta E_{x}$ at a slice $x$ satisfy the linearized version of Eq. (1) with a source noise term for the current $\delta I_{x}$,

$$
\frac{d^{2} \delta E_{x}}{d x^{2}}+P(E) \frac{d \delta E_{x}}{d x}+Q(E) \delta E_{x}=-\frac{\delta I_{x}}{\epsilon A D(E)},
$$

where $^{14}$

$$
\begin{aligned}
& P(E)=\frac{v(E)}{D(E)}, \\
& Q(E)=\frac{D^{\prime}(E)}{D(E)} \frac{d^{2} E}{d x^{2}}+\frac{v^{\prime}(E)}{D(E)}\left(\frac{d E}{d x}-\frac{q}{\epsilon} N_{D}\right) .
\end{aligned}
$$

Here, $\delta I_{x}$ represents the stochastic current induced by the random scattering of carriers inside the semiconductor. It has zero mean and $\delta$-type correlation function $\left\langle\delta I_{x} \delta I_{x^{\prime}}\right\rangle$ $=A K(x) \Delta f \delta\left(x-x^{\prime}\right)$, with $^{17}$

$$
K(x)=4 q^{2} n(x) D(x),
$$

and $\Delta f$ being the frequency bandwidth. Furthermore, at the rectifying metal-semiconductor interface the fluctuations satisfy the linearized version of Eq. (2) with a surface Langevin-like term $\delta I_{0}$. Explicitly,

$$
\left.\frac{d \delta E_{x}}{d x}\right|_{x=0} \equiv \delta E_{0}^{\prime}=-\frac{\delta I_{0}}{\epsilon v_{r}^{0} A} .
$$

Here, $\delta I_{0}$ describes the random nature of the thermionic emission of carriers across the metal-semiconductor interface. It has zero mean and the dispersion $\left\langle\delta I_{0}^{2}\right\rangle=S_{I}^{0} \Delta f$ with $S_{I}^{0}$ given by ${ }^{14}$

$$
S_{I}^{0}=2 q\left[I+2 I_{c}^{0}\right],
$$

and $I_{c}^{0}=q n_{0}^{e q} v_{r}^{0} A$.

At the metal-semiconductor ohmic contact, there is also, in principle, a source of noise of the same nature as the one existing at the rectifying contact. Therefore, at this contact the fluctuations should satisfy 


$$
\left.\frac{d \delta E_{x}}{d x}\right|_{x=L} \equiv \delta E_{L}^{\prime}=\frac{\delta I_{L}}{\epsilon v_{r}^{L} A},
$$

where $\delta I_{L}$ describes the random nature of the thermionic emission of carriers across the ohmic contact. Its correlation function satisfies $\left\langle\delta I_{L}^{2}\right\rangle=S_{I}^{L} \Delta f$, with

$$
S_{I}^{L}=2 q\left[-I+2 I_{c}^{L}\right]
$$

and $I_{c}^{L}=q n_{L}^{e q} v_{r}^{L} A$. Notice the difference in signs between Eqs. (8) and (10), and between Eqs. (9) and (11) because of the opposite current injection at the two contacts.

Equations (4)-(11) constitute a complete set of equations to analyze the noise properties of Schottky barrier diodes in the thermionic-emission-diffusion approximation.

\section{STEADY-STATE SPATIAL PROFILES AND $I-V$ PLOTS}

As it was shown in previous papers, ${ }^{14,16}$ the local and global noise properties of a system are based on the knowledge of the stationary spatial profiles. To find these profiles, in the present case, we solve numerically Eq. (1) with the boundary conditions (2) and (3) by making use of a finite difference scheme.

Before presenting the stationary profiles, let us introduce some characteristic parameters. To this end, we rewrite the boundary conditions in the following form:

$$
\begin{aligned}
& \left.\frac{d E}{d x}\right|_{x=0}=\frac{q N_{D}}{\epsilon}\left(1-\alpha_{0}-\beta J\right), \\
& \left.\frac{d E}{d x}\right|_{x=L}=\frac{q N_{D}}{\epsilon}\left(1-\alpha_{L}+\beta J\right),
\end{aligned}
$$

where $\beta=\mu_{0} E_{\mathrm{th}} / v_{r}^{0}$ is the transport parameter, ${ }^{14} \alpha_{0}$ $=n_{0}^{\mathrm{eq}} / N_{D}$ and $\alpha_{L}=n_{L}^{\mathrm{eq}} / N_{D}$ are the contact parameters, $\mu_{0}$ $=\left.v^{\prime}(E)\right|_{E=0}$ is the low-field mobility, $E_{\mathrm{th}}=k_{B} T /\left(q L_{D}\right)$ the characteristic electric field, and $L_{D}=\left(\epsilon k_{B} T / q^{2} N_{D}\right)^{1 / 2}$ the Debye length. For the sake of simplicity, we have assumed $v_{r}^{0}=v_{r}^{L}$. A slight difference between the recombination velocities of the contacts does not introduce appreciable modifications in the results. Furthermore, the current is normalized as $J=I / I_{R}$, with $I_{R}=q \mu E_{t h} N_{D} A$.

In the following, we take $\beta=1$ assuming that both the thermionic emission and the diffusion of carriers has a comparable effect on the charge transport (for a comparison of the different transport regimes when $\beta$ is varied see Ref. 14). Moreover, we assume $\alpha_{0}=10^{-7}$ and $\alpha_{L}=10$. With these values, for the rectifying contact we have a "high" barrier $\left(\phi_{b}^{0} \approx 26.4 k_{B} T / q \approx 0.68 \mathrm{~V}\right.$ at room temperature) and a “deep" depletion layer $n_{0}^{e q} \sim 10^{-7} N_{D}$. While, for the ohmic contact, we have a "low" barrier $\left(\phi_{b}^{L} \approx 7.9 k_{B} T / q \approx 0.2 \mathrm{~V}\right.$ at room temperature) and a small specific resistance $r_{c}$ $\sim 10^{-6} \Omega \mathrm{cm}^{2}$. For the ohmic contact, the saturation current is $J_{c}^{L}=\alpha_{L} / \beta=10$. For simplicity, we shall consider in the numerical solutions the mobility $\mu$ and the diffusion coefficient $D$ to be constant, although explicit field dependences, characteristic for the hot carrier transport, may be used without additional difficulties. To be concrete, we take $v(E)$ $=\mu E, D=\mu k_{B} T / q$. The analytical solutions, however, will
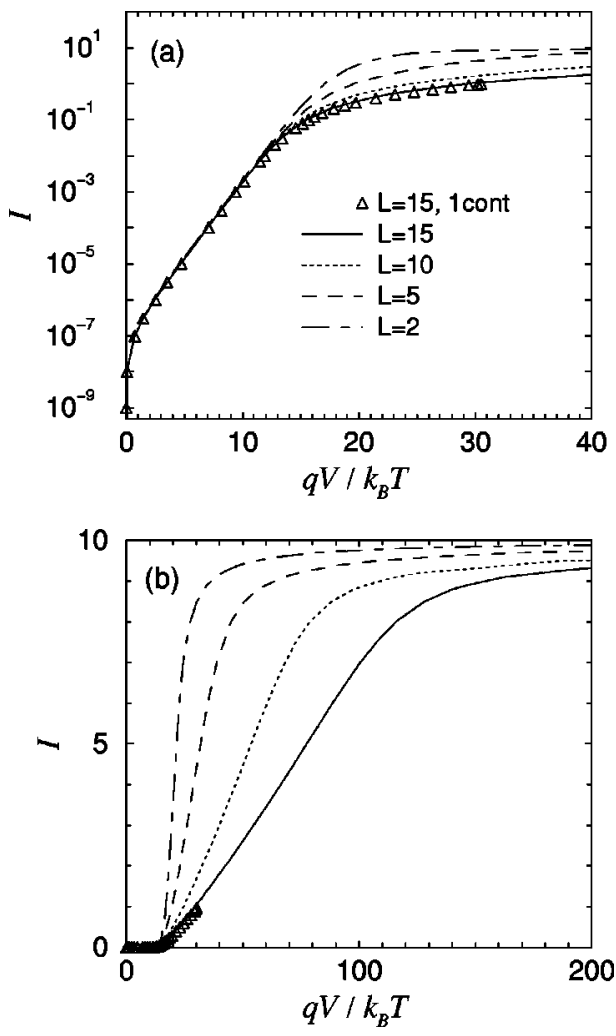

FIG. 1. $I-V$ characteristics for Schottky barrier diodes of different lengths (the current is in units of $I_{R}=q \mu E_{t h} N_{D} A$ ). The results for $L=15 L_{D}$ are compared with those obtained by using flat boundary conditions at the back contact (triangles).

be obtained for the general case when the velocity and diffusion coefficients are field dependent. Finally, we shall consider different epitaxial layer thicknesses: $L / L_{D}=2,5,10$, 15 , which allows us to study the transition from long (micron) to short (submicron) diodes. Note that with the present data $L_{D} \approx 0.13 \mu \mathrm{m}$.

Having found from Eqs. (1)-(3) the electric-field profile $E(x)$ for a given current $J$, the total voltage drop $V$ $=\int_{0}^{L} E(x) d x+\phi_{b}^{0}-\phi_{b}^{L}$, and hence the $I-V$ characteristics, can be easily obtained. The $I-V$ characteristics for the different diode lengths considered, are presented in Fig. 1. For each diode, one can distinguish three different behaviors: (i) an exponential behavior at low voltages, (ii) a linear behavior at intermediate voltages, and (iii) a saturation behavior at high voltages. The exponential behavior can be explained in terms of the thermionic-emission-diffusion theory. ${ }^{18}$ The linear portion of the $I-V$ characteristics shows the existence of series resistance effects. Finally, the saturation of the characteristics at high voltages is a consequence of the nonideality of the ohmic contact, in the present case, a reverse biased metal-semiconductor contact. As is well known, for low barrier heights, the contact behavior is ohmic up to the current values approaching the contact saturation current $J_{c}^{L}$. Other types of ohmic contacts present similar limitations. As can be seen, the value of the epitaxial layer thickness determines the range of voltages covered by each regime: as the epitaxial layer thickness is scaled down, the exponential behavior persists up to higher voltages at the cost of reducing drastically the range of the linear regime. This is equivalent to say 


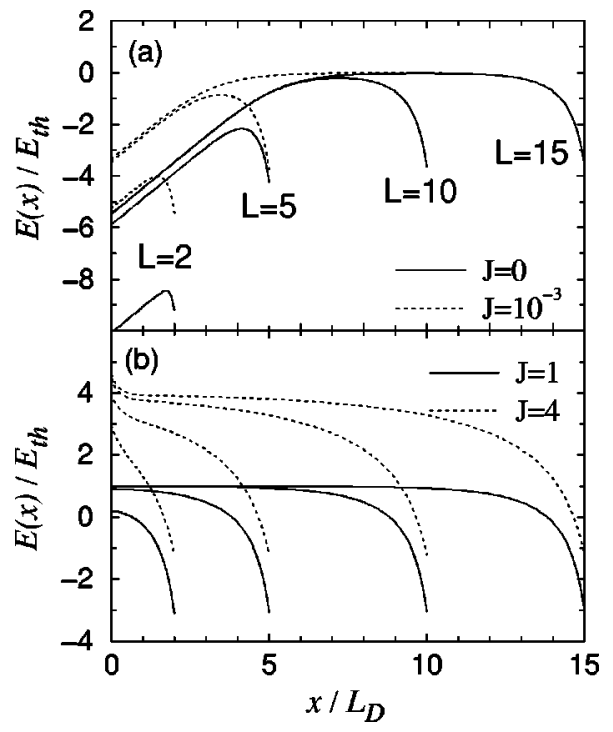

FIG. 2. Stationary electric-field profiles $E(x)$ for Schottky diodes of different lengths $L$, for different currents $J$.

that in this intermediate range the slope of the $I-V$ characteristics increases as the epitaxial layer thickness is decreased. That is, the value of the series resistance, which is proportional to the inverse of the slope of the $I-V$ curve in this regime, is reduced.

This behavior can be easily understood by examining the stationary profiles depicted in Figs. 2 and 3. When the epitaxial layer thickness is reduced, the portion of the sample with constant electric field, which corresponds to a quasineutral region, is reduced, up to vanishing completely for the shortest samples. The absence of the quasineutral region responsible for the series resistance effects explains why the $I-V$ characteristics does not manifest its presence at intermediate voltages.

It is worth mentioning that the $I-V$ characteristics for the diode of length $L=15 L_{D}$ coincides with the one obtained in Ref. 14 (triangles), where a simplified model with flat

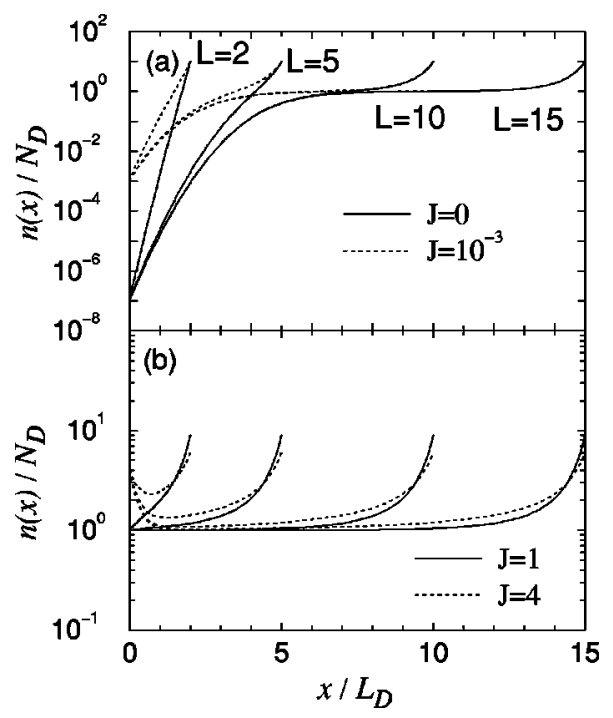

FIG. 3. Stationary electron-density profiles $n(x)$ for Schottky diodes of different lengths $L$, for different currents $J$. boundary conditions at the back ohmic contact was considered. The same correspondence is observed for the stationary profiles except for a narrow layer nearby the ohmic contact, which has no effect on the $I-V$ characteristics. These results confirm that the simple approach developed in Ref. 14 is valid to deal with long diodes. The same conclusion will be taken below for the noise properties.

Finally, we note that when the realistic ohmic contact (with nonzero barrier height) is considered, the electric field profile displays a maximum in a certain point inside the diode [Fig. 2(a)] or an inflexion point [Fig. 2(b)]. It is seen from Eqs. (12), that the maximum appears when the current is below the so-called flatband current value $J^{*}=(1$ $\left.-\alpha_{0}\right) / \beta \approx 1$ with the present data. The presence of this maximum makes necessary to modificate the mathematical treatment of Ref. 14 for the analysis of the noise characteristics, as it will be discussed below. In the following, two different current regimes will be considered: (i) low currents, $0<J<J^{*}$, when the electric-field displays a maximum [Fig. 2(a)], and (ii) high currents, $J>J^{*}$, when the electric-field displays an inflection point with $d^{2} E / d x^{2}=0$, or $d n / d x=0$ [Figs. 2(b), 3(b)].

\section{IMPEDANCE AND NOISE}

\section{A. Analytical solution}

To characterize the noise properties of a Schottky diode, we have to solve a second-order stochastic differential Eq. (4) with space dependent coefficients [this space dependence comes through the space dependence of the electric field $E(x)]$. To find the solution, we use a recently developed analytical method ${ }^{16}$ which has been successfully applied to the case of an $n^{+} n$ junction ${ }^{16}$ and a Schottky barrier contact. ${ }^{14}$ By means of this method, an explicit expression for the fluctuation of the electric field $\delta E_{x}$, which solves Eq. (4) and satisfies the boundary conditions (8) and (10), has been derived (see Appendix A for the details). The fluctuation of the voltage between the contacts can then be computed as a sum of three contributions

$$
\delta V=Z_{0} \delta I_{0}+Z_{L} \delta I_{L}+\int_{0}^{L} \nabla Z(x) \delta I_{x} d x .
$$

The last term in Eq. (13) gives the contribution to the voltage fluctuation from the bulk of the diode. Each local noise contribution is characterized by the bulk impedance field $\nabla Z(x)$. Moreover, $Z_{0}$ and $Z_{L}$ are the impedances of the rectifying and back ohmic contacts, respectively. They are given by (compare with Eq. (A5) in Appendix A)

$$
Z_{0}=\frac{E_{0}-E_{\Delta}}{\epsilon v_{r}^{0} A \rho_{0}^{\prime}}, \quad Z_{L}=\frac{E_{L}-E_{\Delta}}{\epsilon v_{r}^{L} A \rho_{L}^{\prime}},
$$

where $\rho_{0, L}^{\prime}=E_{0, L}^{\prime \prime}$. The expressions for $\nabla Z(x)$, as well as for the parameter $E_{\Delta}$ entered in Eq. (14), have been derived in Appendix A in a general form. Below, we present them in a form more suitable for computations, which take into account the features of the electric-field profiles $E(x)$. As was 
pointed out in Sec. III, according to the value of the current, two different steady-state transport regimes may be distinguished.

(i) Low currents, $0<J<J^{*}$. This current regime is characterized by the presence of a depletion layer near the rectifying contact, and a relatively small accumulation layer near the back ohmic contact [Fig. 3(a)]. Accordingly, the electric field displays a maximum in a certain point inside the diode [Fig. 2(a)]. For this case, the following expressions should be used

$$
\begin{aligned}
& \nabla Z(x)=\frac{\rho(x)}{\psi(x)} \int_{0}^{x}\left\{\left[E_{\Delta}-E(\xi)\right] \frac{Q(\xi)}{\rho^{\prime}(\xi)}-1\right\} \frac{W(\xi)}{\rho^{\prime}(\xi)} d \xi \\
& +\frac{W(x)}{\rho^{\prime}(x) \psi(x)}\left[E(x)-E_{\Delta}\right], \\
& E_{\Delta}=\frac{\int_{0}^{L}\left[1+\frac{E(x) Q(x)}{\rho^{\prime}(x)}\right] \frac{W(x)}{\rho^{\prime}(x)} d x}{\int_{0}^{L} \frac{Q(x) W(x)}{\left[\rho^{\prime}(x)\right]^{2}} d x} .
\end{aligned}
$$

(ii) High currents, $J>J^{*}$. For this current regime, no depletion layer appears, instead, two accumulation layers at the both contacts are observed [Fig. 3(b)]. Accordingly, the electric field displays an inflection point where $d^{2} E / d x^{2}=0$ [Fig. 2(b)]. For this case, the following expressions are valid:

$$
\begin{gathered}
\nabla Z(x)=\frac{\rho(x)}{\psi(x)}\left[\int_{0}^{x}\left[E_{\Delta}-E(\xi)\right] \frac{W(\xi)}{\rho^{2}(\xi)} d \xi\right. \\
\left.+\frac{W_{0}}{\rho_{0} \rho_{0}^{\prime}}\left(E_{0}-E_{\Delta}\right)\right], \\
E_{\Delta}=\frac{\int_{0}^{L} \frac{E(x) W(x)}{\rho^{2}(x)} d x+\frac{E_{L} W_{L}}{\rho_{L} \rho_{L}^{\prime}}-\frac{E_{0} W_{0}}{\rho_{0} \rho_{0}^{\prime}}}{\int_{0}^{L} \frac{W(x)}{\rho^{2}(x)} d x+\frac{W_{L}}{\rho_{L} \rho_{L}^{\prime}}-\frac{W_{0}}{\rho_{0} \rho_{0}^{\prime}}} .
\end{gathered}
$$

In Eqs. (15)-(18) we have used the same notations as in Refs. 14, 16. Namely, $\rho(x)=d E / d x, \psi(x)=\epsilon A D(x) W(x)$, and $W(x)=W_{0} \exp \left\{-\int_{0}^{x} v[E(\xi)] / D[E(\xi)] d \xi\right\}$. The function $Q$ in Eqs. (15)-(16) is determined by Eq. (6).

Now we can determine the total impedance $Z$ of the diode

$$
Z=Z_{0}+Z_{L}+\int_{0}^{L} \nabla Z(x) d x \equiv Z_{0}+Z_{L}+Z_{b},
$$

the spectral density of voltage fluctuations

$$
\begin{aligned}
S_{V} & =Z_{0}^{2} S_{I}^{0}+Z_{L}^{2} S_{I}^{L}+\int_{0}^{L}[\nabla Z(x)]^{2} K(x) d x \\
& \equiv S_{V 0}+S_{V \mathrm{~L}}+S_{V \mathrm{~b}},
\end{aligned}
$$

and the spectral density of current fluctuations

$$
S_{I}=\frac{S_{V 0}+S_{V L}+S_{V b}}{\left(Z_{0}+Z_{L}+Z_{b}\right)^{2}} .
$$

In the previous expressions, $Z_{b}$ stands for the impedance of the bulk part of the diode. Moreover, $S_{V 0}, S_{V L}$, and $S_{V b}$ refer to the contribution to the voltage fluctuations coming from the rectifying contact, the ohmic contact, and the diode bulk, respectively. Finally, the noise temperature takes on the form

$$
T_{n}=\frac{1}{4 k_{B}} \frac{S_{V 0}+S_{V L}+S_{V b}}{Z_{0}+Z_{L}+Z_{b}} .
$$

Equations (19)-(23) constitute the analytical expressions for the net impedance and noise characteristics of a Schottky diode as obtained from the thermionic-emission-diffusion theory. Furthermore, they also give information about the spatial distribution of the impedance throughout the device by means of the impedance field $\nabla Z(x)$ and the local contributions to the voltage fluctuations by virtue of the spatial profile $s_{V}(x)=[\nabla Z(x)]^{2} K(x)$. These local distributions may be very useful in interpreting the results as will be seen in what follows. It is worth noting that in order to compute the impedance and noise characteristics, we only need to know the stationary electric field profile $E(x)$.

It is important to see the connection between the presented formulas for the Schottky diode (with a realistic consideration of a back ohmic contact) with the formulas when the flat boundary conditions at the back contact are imposed

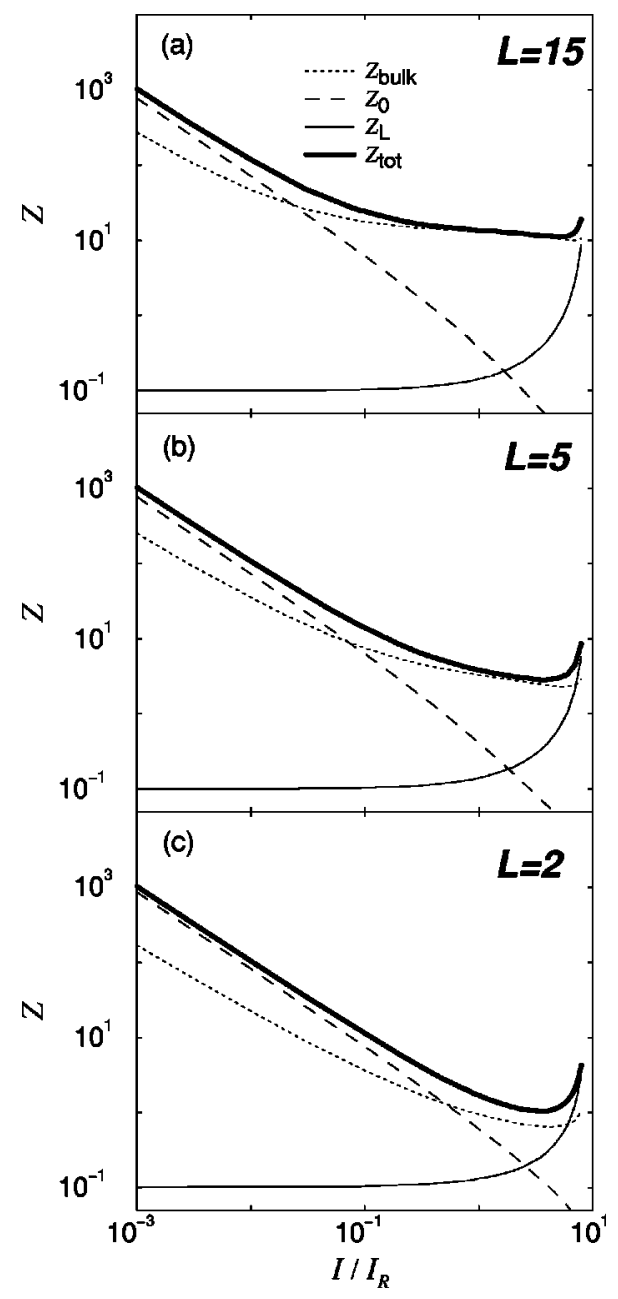

FIG. 4. Impedance $Z$ vs current $I$ for different diode lengths $L$ : (a) $L$ $=15 L_{D}$, (b) $L=5 L_{D}$, and (c) $L=2 L_{D}$. The relative contributions from the contacts $Z_{0}$ and $Z_{L}$, and from the bulk $Z_{b}$ are compared (all impedances are normalized by $\left.R_{R}=L_{D} / q \mu N_{D} A\right)$. 


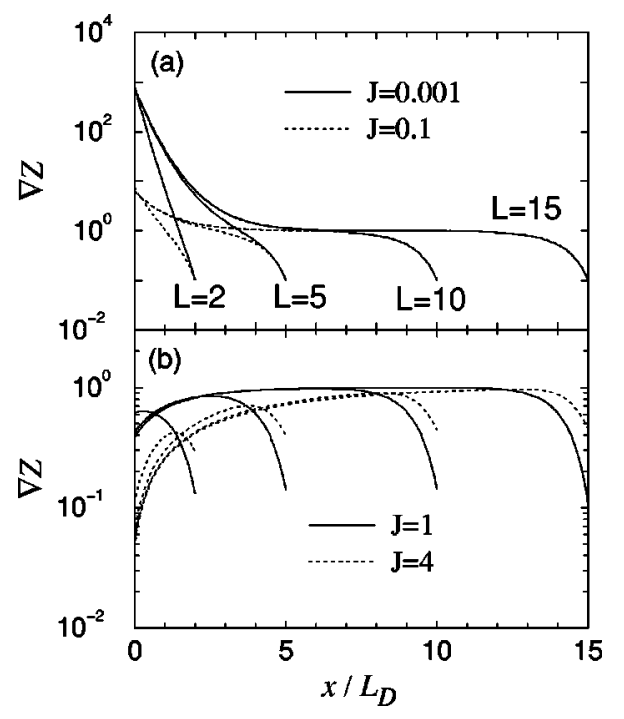

FIG. 5. Spatial profiles for the local impedance $\nabla Z(x)$ [normalized by $\left.\left(q \mu N_{D} A\right)^{-1}\right]$ for different currents $J$ and diode lengths $L$.

(valid for long diodes with $L \gg L_{D}$ ). In the latter case, due to the flat conditions at $x=L$ one can take $\rho_{L} \rightarrow 0, E_{\Delta} \rightarrow E_{L}$, and the electric-field profile does not display neither a maximum nor an inflection point. ${ }^{14}$ Therefore, one can use any of the two sets of equations for the impedance field. In particular, in Ref. 14 we used Eqs. (17) and (18).

\section{B. Results}

In this subsection, we shall evaluate the analytical expressions derived in the previous section. For low currents, when the main current limiting process comes from the depletion layer, simplified formulas can be used, as shown in Appendix B.

In Fig. 4 we present the total impedance $Z$ as a function of the current for different diode lengths. We may distinguish here clearly the same current regimes as observed earlier for the $I-V$ characteristics: (i) a low current regime, in which $Z$ decreases with the current, (ii) an intermediate current regime, in which $Z$ is nearly constant, and (iii) a high current regime, in which $Z$ increases sharply with the current. As can be seen in Fig. 4 in the low current regime, the main contributions to the impedance comes from $Z_{b}$ and $Z_{0}$, in the intermediate current regime from $Z_{b}$, and in the high current regime from $Z_{L}$. The relative importance of the spatial contributions to $Z_{b}$ also depends on the current regime considered. As shown in Fig. 5, where the impedance field $\nabla Z(x)$ is plotted, for low currents the main contribution to $Z_{b}$ comes from the region near the rectifying contact, while for intermediate and high currents, it comes basically from the "central" part of the diode.

It should be pointed out that the range of currents covered by each current regime depends appreciably on the diode size. When the epitaxial layer thickness is scaled down, the low current range extends towards higher currents at the cost of reducing the intermediate current range. The value of the impedance in this intermediate regime then decreases, that can be easily understood in terms of the progressive

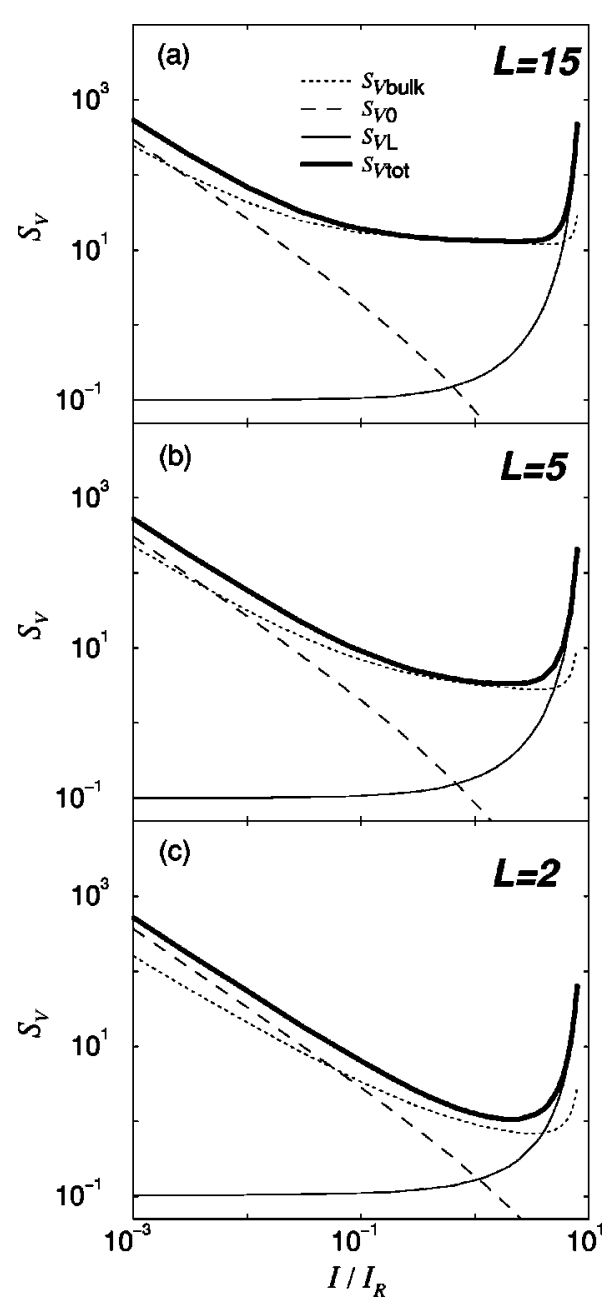

FIG. 6. Voltage fluctuations $S_{V}$ vs current $I$ for different diode lengths $L$ : (a) $L=15 L_{D}$, (b) $L=5 L_{D}$, and (c) $L=2 L_{D}$. The relative contributions from the contacts $S_{V 0}$ and $S_{V L}$, and from the bulk $S_{V b}$ are compared (all normalized by $4 k_{B} T L_{D} / q \mu N_{D} A$ ).

reduction of the series resistance effects. The high current regime ends up at the ohmic-contact saturation current $J_{L}^{c}$, which is independent of the diode size.

A similar analysis has been performed for the spectral density of voltage fluctuations. The relative significance of different contributions is shown in Fig. 6 for different diode lengths. As before, one may distinguish three different current regimes: (i) at low currents, $S_{V}$ decreases with the current and the main contributions are due to $S_{V 0}$ and $S_{V b}$, (ii) at intermediate currents, $S_{V}$ is almost constant and $S_{V b}$ dominates, and (iii) at high currents, $S_{V}$ increases sharply and the main contribution comes from $S_{V L}$. Notice that the ranges of these three current regimes do not correspond directly to those found for the impedance and the $I-V$ characteristics. Indeed, in Fig. 6 it can be observed that the bulk contribution to the voltage fluctuations becomes more important at lower currents than the bulk contribution to the impedance in Fig. 4. This fact can be understood in terms of the existence of spatial crosscorrelations inside the diode. ${ }^{14}$

The local contributions to the voltage fluctuations $S_{V b}$ are shown in Fig. 7 by virtue of the spatial profiles $s_{V}(x)$ $=[\nabla Z(x)]^{2} K(x)$. For low currents, the main contribution 


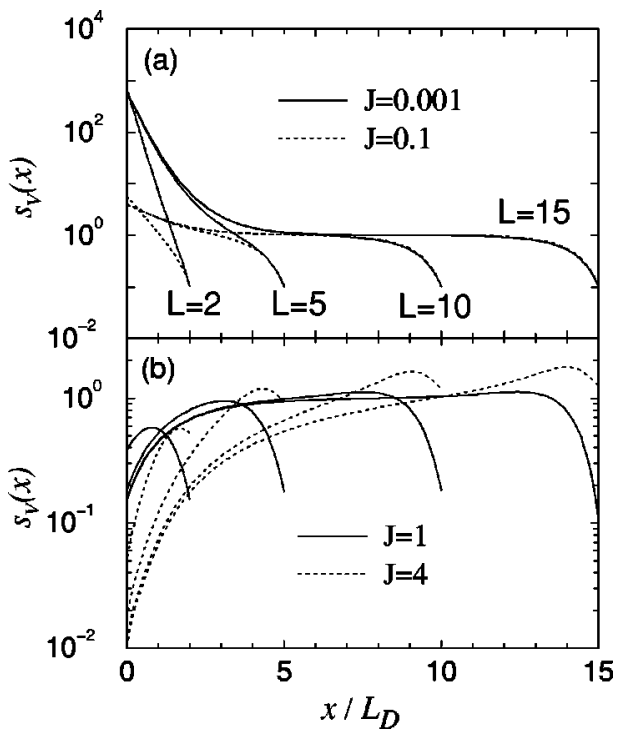

FIG. 7. Spatial profiles for the local noise distribution $s_{V}(x)$ [normalized by $\left.4 k_{B} T \Delta f / \mu N_{D} A\right]$ for different currents $J$ and diode lengths $L$.

comes from the region near the rectifying contact (depletion layer), while at intermediate and high currents, $S_{V b}$ receives contributions from the "central" part of the diode and from the ohmic contact region.

Having found the total impedance $Z$ and the voltage noise spectral density $S_{V}$, the spectral density of current fluctuations $S_{I}$ can be computed from Eq. (22). The results as a function of the current for the different diode lengths are shown in Fig. 8. At very low currents, $S_{I}$ is constant, since it gives the thermal equilibrium noise. Then exhibits a $2 q I$ shot-noise-like behavior. These two regimes closely follow the law $S_{I}=2 q I \operatorname{coth}\left(q V / 2 k_{B} T\right)$, which has been discussed in detail in Ref. 14. In the intermediate current regime, $S_{I}$ deviates from this law and tends to saturate, due to the presence of series resistance effects. As the length of the diode is scaled down, this saturation progressively disappears. Finally, in the high current regime $S_{I}$ increases sharply when the ohmic contact saturation current is approached.

The effective noise temperature $T_{n}$ vs current is depicted in Fig. 9 for different diode lengths. In the low current re-

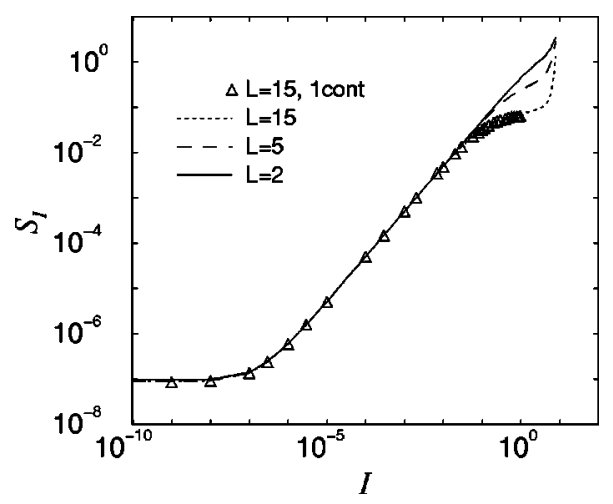

FIG. 8. Current-noise spectral density $S_{I}$ vs current $I$ for different diode lengths $L$. The results for $L=15 L_{D}$ are shown to coincide with those obtained with flat boundary conditions at the back contact (triangles). $S_{I}$ and $I$ are normalized by $4 q I_{R}$ and $I_{R}=q \mu E_{t h} N_{D} A$, respectively.

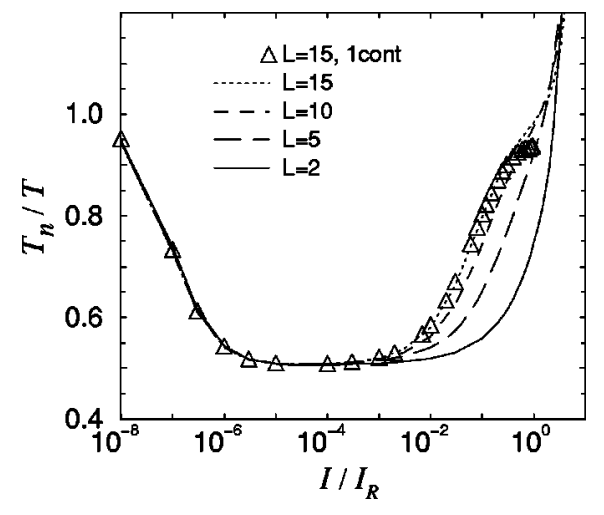

FIG. 9. Noise temperature $T_{n}$ vs current $I$ for different diode lengths $L$. The results for $L=15 L_{D}$ are compared with those obtained with flat boundary conditions at the back contact (triangles).

gime, the noise temperature starts from the lattice temperature $T_{n}=T$, and then drops towards the $T / 2$ value, that is a consequence of the $2 q I$ law and is typical of the shot-noise behavior. ${ }^{14}$ For intermediate currents $T_{n}$ increases again, tending to saturate around the ambient temperature $T$, where the diffusion noise of a passive resistor dominates. For the shortest samples, this intermediate regime is not displayed, due to the small value of the series resistance. Finally, in the high current regime, $T_{n}$ increases sharply when the ohmiccontact saturation current is reached. As mentioned before, the range covered for each current regime is size dependent. Indeed, by reducing the epitaxial layer thickness, the noise temperature keeps its shot-noise-like behavior up to higher currents, leading to the noise reduction. This improvement in noise temperature may be, however, limited by the ohmic contact. This occurs when the current approaches the ohmiccontact saturation current. Then, a further decrease in the epitaxial layer thickness does not give rise to a further appreciable improvement in the noise temperature. In a such situation, the device performance can only be improved by improving the quality of the ohmic contact.

\section{CONCLUSIONS}

In the present article, we have presented a theory to describe the combined effects of the epitaxial layer thickness and the back ohmic contact on the noise properties of Schottky barrier diodes. The theory is formulated under the thermionic-emission-diffusion framework and is applicable in the whole range of biases. By means of this theory we have shown that the value of the epitaxial layer thickness has considerable effects on both the current operation regime and the noise properties of these devices. To this respect, one can see that when the epitaxial layer thickness is scaled down, the range of currents in which the shot-noise-like behavior is displayed increases at the cost of reducing the current range dominated by the bulk thermal-noise-like behavior. For thin epitaxial layers, this last current range is almost suppressed, with the consequent reduction in the noise temperature. This noise reduction is limited, however, by the nonideality of the back ohmic contact. Indeed, when the current approaches the ohmic-contact saturation current, a further reduction in the epi-layer thickness does not give rise to an appreciable re- 
duction in the noise temperature. In such a case, one needs to improve the quality of the ohmic contact in order to improve the device performance. It is worth noting that many of these phenomena might be present in THz Schottky barrier diodes used for mm-wave and sub-mm-wave applications, which are designed with rather thin epitaxial layer and operate at intermediate or high currents.

Finally, it should be pointed out that the theoretical procedure presented in this article is not only restricted to the study of Schottky barrier diodes, but it can be easily extended to the study of other devices. Our work then opens new perspectives on what concerns the noise analysis of semiconductor devices operating under strongly inhomogeneous distributions of the electric field and charge concentrations.

\section{APPENDIX A: IMPEDANCE FIELD OF A SCHOTTKY DIODE: GENERAL EXPRESSIONS}

The general scheme to solve the stochastic Eq. (4) has been outlined in Ref. 16. The main idea is to find two linearly independent solutions of the corresponding homogeneous equation and then to use them to construct the solution of the nonhomogeneous Eq. (4) satisfying the appropriate boundary conditions. One of these particular solutions is $\rho(x)=d E / d x$. The second solution may be expressed through the first one, but its form depends on the properties of the function $\rho$ (i.e., on the features of the electric-field profile in the system under consideration). If $\rho(x) \neq 0[E(x)$ does not display a maximum or a minimum], one can use the following expression for the second function: ${ }^{16}$

$$
u(x)=\rho(x) \int_{C}^{x} \frac{W(\xi)}{[\rho(\xi)]^{2}} d \xi
$$

Otherwise, one can use the alternative expression

$$
u(x)=-\frac{W(x)}{\rho^{\prime}(x)}+\rho(x) \int_{C}^{x} \frac{Q(\xi) W(\xi)}{\left[\rho^{\prime}(\xi)\right]^{2}} d \xi,
$$

under the condition that $\rho^{\prime}(x) \neq 0[E(x)$ does not display an inflection point]. The function $Q$ in the last formula is determined by Eq. (6). If $E(x)$ displays neither a maximum (or a minimum) nor an inflection point, both expressions for $u(x)$ are equivalent.

By means of these functions, the general solution of Eq. (4) reads $^{14}$

$$
\begin{aligned}
\delta E_{x}= & C_{1} \rho(x)+C_{2} u(x)+\rho(x) \int_{0}^{x} \frac{u(\xi)}{\psi(\xi)} \delta I_{\xi} d \xi \\
& +u(x) \int_{x}^{L} \frac{\rho(\xi)}{\psi(\xi)} \delta I_{\xi} d \xi .
\end{aligned}
$$

The derivatives of the fluctuation of the electric field at the boundaries $\delta E_{0}^{\prime}, \delta E_{L}^{\prime}$ are known from Eqs. (8) and (10). They can be used to eliminate the constants $C_{1}, C_{2}$, one gets

$$
\begin{aligned}
\delta E_{x}= & \frac{1}{\Delta}\left[\frac{\delta E_{0}^{\prime}}{\rho_{0}^{\prime}}-\frac{u_{0}^{\prime}}{\rho_{0}^{\prime}} \int_{0}^{L} \frac{\rho(\xi)}{\psi(\xi)} \delta I_{\xi} d \xi\right]\left[\frac{u_{L}^{\prime}}{\rho_{L}^{\prime}} \rho(x)-u(x)\right] \\
& +\frac{1}{\Delta}\left[-\frac{\delta E_{L}^{\prime}}{\rho_{L}^{\prime}}+\int_{0}^{L} \frac{u(\xi)}{\psi(\xi)} \delta I_{\xi} d \xi\right]\left[\frac{u_{0}^{\prime}}{\rho_{0}^{\prime}} \rho(x)-u(x)\right] \\
& +\rho(x) \int_{0}^{x} \frac{u(\xi)}{\psi(\xi)} \delta I_{\xi} d \xi+u(x) \int_{x}^{L} \frac{\rho(\xi)}{\psi(\xi)} \delta I_{\xi} d \xi,
\end{aligned}
$$

where $\Delta=\left(u_{L}^{\prime} / \rho_{L}^{\prime}\right)-\left(u_{0}^{\prime} / \rho_{0}^{\prime}\right)$, and prime in all the functions stands for the derivative. Equation (A4) determines the stochastic value of the electric field $\delta E_{x}$ at any slice $x$ from the noise sources $\delta I_{x}$ and the stochastic boundary conditions $\delta E_{0}^{\prime}$ and $\delta E_{L}^{\prime}$. Integrating this equation, one obtains the voltage fluctuation as a sum of three contributions

$$
\delta V=\frac{E_{0}-E_{\Delta}}{\epsilon v_{r}^{0} A \rho_{0}^{\prime}} \delta I_{0}+\frac{E_{L}-E_{\Delta}}{\epsilon v_{r}^{L} A \rho_{L}^{\prime}} \delta I_{L}+\int_{0}^{L} \nabla Z(x) \delta I_{x} d x,
$$

where we have introduced the parameter having a dimension of the electric field

$$
E_{\Delta}=\frac{1}{\Delta}\left[\frac{u_{L}^{\prime} E_{L}}{\rho_{L}^{\prime}}-\frac{u_{0}^{\prime} E_{0}}{\rho_{0}^{\prime}}-\int_{0}^{L} u(x) d x\right] .
$$

The first two terms in the rhs of Eq. (A5) determine the impedances of the rectifying and back ohmic contacts, whereas the last term gives the bulk impedance field in the form

$$
\begin{aligned}
\nabla Z(x)= & \frac{\rho(x)}{\psi(x)}\left[\left(E_{0}-E_{\Delta}\right) \frac{u_{0}^{\prime}}{\rho_{0}^{\prime}}+\int_{0}^{x} u(\xi) d \xi\right] \\
& +\left[E_{\Delta}-E(x)\right] \frac{u(x)}{\psi(x)} .
\end{aligned}
$$

The expressions (A6) and (A7) for $E_{\Delta}$ and $\nabla Z(x)$ are written in a general form in terms of the auxiliary functions $\rho$ and $u$. By further substituting either Eq. (A1) or (A2) for $u(x)$, one arrives to Eqs. (17) and (18) or Eqs. (15) and (16), respectively. Finally, the expressions for the contact impedances $Z_{0}$ and $Z_{L}$ in Eqs. (14) can be obtained directly from Eq. (A5).

\section{APPENDIX B: IMPEDANCE FIELD FOR $0<\boldsymbol{J} \ll \boldsymbol{J} *$}

The expression for the impedance field for the current regime corresponding to $0<J \ll J^{*}$ can be simplified. This is the situation when the depletion layer dominates the bulk resistance of the diode. It turns out, that the integral in the rhs of Eq. (15) and the characteristic field $E_{\Delta}$ are negligibly small (they are of the order of the current $J \ll 1$ ). At equilibrium they vanish identically. Therefore, for this current regime one can approximate

$$
\nabla Z(x) \approx \frac{W(x) E(x)}{\rho^{\prime}(x) \psi(x)} \approx \frac{1}{q \mu[E(x)] n(x) A},
$$

where $\mu(E)$ is the field-dependent mobility of the semiconductor. In this equation, the impedance field is just the local (per unit length) resistance, as it occurs at equilibrium. 
${ }^{1}$ T. W. Crowe, R. J. Mattauch, H. P. Röser, W. L. Bishop, W. C. B. Peatman, and X. Liu, Proc. IEEE 80, 1827 (1992).

${ }^{2}$ U. V. Bhapkar and T. W. Crowe, IEEE Trans. Microwave Theory Tech. 40, 886 (1992).

${ }^{3}$ A. Jelenski, A. Grüb, V. Krozer, and H. L. Hartnagel, IEEE Trans. Microwave Theory Tech. 41, 549 (1993).

${ }^{4}$ V. Krozer and A. Grüb, Solid-State Electron. 37, 169 (1994).

${ }^{5}$ P. Marsh, D. Pavlidis, and K. Hong, IEEE Trans. Electron Devices 45, 349 (1998).

${ }^{6}$ B. L. Gelmont, D. L. Woolard, J. L. Hesler, and T. W. Crowe, IEEE Trans. Electron Devices 45, 2521 (1998).

${ }^{7}$ J. G. Adams, A. Jelenski, D. H. Navon, and T. W. Tang, IEEE Trans. Electron Devices ED-34, 1963 (1987).

${ }^{8}$ A. van der Ziel, Noise in Solid State Devices and Circuits (Wiley, New York, 1986)

${ }^{9}$ T. Viola and R. Mattauch, J. Appl. Phys. 44, 2805 (1973).
${ }^{10}$ G. M. Hegazi, A. Jelenski, and K. S. Yngvesson, IEEE Trans. Microwave Theory Tech. 33, 1404 (1985).

${ }^{11}$ H. Zirath, J. Appl. Phys. 60, 1399 (1986).

${ }^{12}$ M. Trippe, G. Bosman, and A. van der Ziel, IEEE Trans. Microwave Theory Tech. 34, 1183 (1986).

${ }^{13}$ A. Jelenski, E. L. Kollberg, and H. H. G. Zirath, IEEE Trans. Microwave Theory Tech. 34, 1193 (1986).

${ }^{14}$ G. Gomila, O. M. Bulashenko, and J. M. Rubí, J. Appl. Phys. 83, 2619 (1998).

${ }^{15}$ T. González, D. Pardo, L. Varani, and L. Reggiani, Appl. Phys. Lett. 63, 3040 (1993); J. Appl. Phys. 82, 2349 (1997).

${ }^{16}$ O. M. Bulashenko, G. Gomila, J. M. Rubí, and V. A. Kochelap, Appl. Phys. Lett. 70, 3248 (1997); J. Appl. Phys. 83, 2610 (1998).

${ }^{17}$ C. M. van Vliet, IEEE Trans. Electron Devices 41, 1902 (1994).

${ }^{18}$ E. H. Rhoderick and R. H. Williams, Metal-Semiconductor Contacts, 2nd ed. (Clarendon Press, Oxford, 1988). 